\title{
Effective demagnetizing tensors in arrays of magnetic nanopillars
}

\author{
P. Mendoza Zélis, ${ }^{1,2}$ V. Vega,${ }^{3}$ V. M. Prida, ${ }^{3}$ L. C. Costa-Arzuza, ${ }^{4}$ F. Béron, ${ }^{5}$ \\ K. R. Pirota, ${ }^{5}$ R. López-Ruiz, ${ }^{5, *}$ and F. H. Sánchez ${ }^{1, \dagger}$ \\ ${ }^{1}$ IFLP, CCT La Plata, CONICET and Departamento de Física, Facultad de Ciencias Exactas, C. C. 67, \\ Universidad Nacional de La Plata, 1900 La Plata, Argentina \\ ${ }^{2}$ Departamento de Ciencias Básicas, Facultad de Ingeniería, Universidad Nacional de La Plata, 1900 La Plata, Argentina \\ ${ }^{3}$ Departamento de Física - Universidad de Oviedo, C/Federico Garcia Lorca n 18, Oviedo 33007, Spain \\ ${ }^{4}$ Departamento de Ciencias Naturales y Exactas - Universidad de la Costa C/ 58 No. 55-66, Barranquilla 080002, Colombia \\ ${ }^{5}$ Instituto de Física Gleb Wataghin - Universidade Estadual de Campinas (UNICAMP), Campinas (SP) 13083-859, Brazil \\ (Received 5 June 2017; revised manuscript received 25 October 2017; published 21 November 2017)
}

\begin{abstract}
A model describing the effect of magnetic dipolar interactions on the susceptibility of magnetic nanopillars is presented. It is an extension of a recently reported model for three-dimensional randomlike dispersions of nearly spherical nanoparticles in equilibrium [Sánchez et al., Phys. Rev. B 95, 134421 (2017)], to well-ordered arrays of nanoparticles out of equilibrium. To test it, a high-quality benchmark consisting of a two-dimensional hexagonal arrangement of quasi-identical parallel nickel nanopillars embedded in a porous alumina template was fabricated. This model is based on an effective demagnetizing tensor, which only depends on a few morphological parameters of the sample, as the nearest-neighbor distance between pillars and the volume fraction of pillars in the specimen. It allows us to obtain the nanopillar intrinsic susceptibility tensor from the magnetic response of the nanopillar ensemble. The values of the in-plane and normal-to-plane susceptibility of the sample are successfully predicted by the model. Furthermore, the model reproduces the susceptibility in the applied field direction, measured for different applied field angles. In this way, it provides a simple and accurate treatment to account for the complex magnetic effects produced by dipolar interactions.
\end{abstract}

DOI: 10.1103/PhysRevB.96.174427

\section{INTRODUCTION}

Magnetic particles in dense arrangements interact intensely [1]. Some literature even reports on dipolar interactions as responsible for possible phase transitions to collective longrange magnetic ordering states such as spin glasses [2], an issue still controversial [3]. Dipolar interactions have two remarkable effects on the magnetic response of a nanoparticle ensemble. On one hand they alter relaxation mechanisms, affecting time response, including moment blocking. On the other hand, the dipolar field adds to the external applied field, giving rise to susceptibility apparent changes, even in thermal equilibrium. The study of this second effect in a controlled environment is the objective of the present paper. Solid close-packed assemblies are optimal environments because of the accurate experimental control of the two involved geometric features, namely, the particle morphology and the particles ordering arrangement [4]. The anodic aluminum oxide, extensively used for nanowires production, provides a good patterned template to design these assemblies [5-7]. In systems with low or negligible magnetocrystalline anisotropy, the magnetic behavior is determined by the morphology and the spatial distribution of the particles, via shape anisotropy and dipolar interactions, respectively. From the experimental point of view, it is observed in nanowire ordered arrays having low magnetocrystalline anisotropy, that the magnetic response varies between two limits: the easy magnetization axis parallel to wires (wire anisotropy dominates) or parallel to the film plane (interwire dipolar effects dominate) $[5,8]$. This magnetic

\footnotetext{
*rlruiz@ifi.unicamp.br

†sanchez@ fisica.unlp.edu.ar
}

behavior is often evaluated by the competition of two types of effects: (i) intrinsic, due to individual wire properties and (ii) extrinsic, due to the wire ensemble characteristics, in particular the volume fraction occupied by the wires [9-12]. From the applied point of view, the quantification of these intrinsic and extrinsic contributions is of great importance. For instance, in perpendicular recording media, the intrinsic switching field distribution of the bites constitutes the main quality parameter [13], and for hyperthermia applications, extrinsic effects can both reduce or increase the treatment efficiency [14,15]. Thus, it is important to obtain models and procedures to foresee and determine the dipolar effects, isolating the intrinsic behavior from the response of the ensemble.

In ensembles of nanowires, dipolar interactions produce the shift of the switching field of the wires, producing a sheared hysteresis loop. Bearing in mind that due to size distribution, misalignments or impurities, there is no unique switching field, i.e., the hysteresis loop of the ensemble is not squared even in the absence of interactions, the difficulty consists in quantifying the extent of the dipolar interactions in the experimental response of the ensemble. The main experimental methods in order to give account of this extrinsic dipolar influence are based on the quantification of the Wolfarth relation deviation using isothermal and dc-demagnetizing remanence measurements, via Henkel or $\delta M$ plots $[16,17]$. The main restriction is the need of a high-remanence system. The reversibility analysis of minor hysteresis loops, sometimes complex in the case of first-order reversal curve (FORC) diagrams [18], is also used for this purpose [19]. For instance, $\delta H$ method is largely used [20,21], but presupposes the analytical form of the intrinsic switching field distribution. Local in-field imaging measurements give experimental access to the intrinsic response of the system [22]. Unfortunately, 
these are yet difficult and time-consuming measurements, especially working at low temperatures.

In the present paper, a simple and practical model for the effective demagnetizing tensor (EDT), which allows the determination of the intrinsic and extrinsic effects on the susceptibility of two-dimensional ordered arrangements of nanopillars (NPs) out of equilibrium, is presented. The tensor description is needed because the problem is, in general, anisotropic. In the present case, this becomes evident since the anisotropic particles (nanopillars) are oriented along a specific direction. Thus, an EDT model for discontinuous and ordered magnetic materials is required. This model extends the EDT concept proposed by Sanchez et al. [23], initially applied to three-dimensional randomlike dispersions of magnetic single-domain particles in thermal equilibrium, to a two-dimensional ordered arrangement out of equilibrium. As it will be shown, the proposed EDT model depends on the nearest-neighbor distance between NPs relative to NPs diameter, the associated volume fraction of pillars in the specimen, and the demagnetizing tensor corresponding to the specimen shape. The main gain is that the only requisite is the morphological characterization of the ensemble, very accessible by standard imaging techniques. Furthermore, the tensor formalism allows us to determine the intrinsic susceptibility at any direction in an easy and rapid way. It is not necessary (i) any treatment of remanence measurements (only applicable in case of high-remanence systems) [16,17] nor (ii) the reversibility analysis of minor loops (qualitative in the case of FORC diagrams [18]) supposing the functional form of the intrinsic switching field distribution and neglecting reversible processes [19-21].

The present model improves previous attempts to describe and quantify the effects of dipolar interaction on nanoparticle assembly susceptibility. Some phenomenological models use parameters introduced without sufficient justification (see Ref. [24] for examples), such as temperature [25] or field additive parameters [5]. Although they have been successfully applied, their parameters do not provide information on the specimen structure. The present EDT model is simple, predictive, and allows the determination of some important details about the specimen geometry and its internal configuration.

\section{SYNTHESIS AND STRUCTURE OF NICKEL NP ARRAY}

Nickel NPs were fabricated via dc electrodeposition within the nanopores of porous alumina membrane. This membrane was grown through mild anodization process of high-purity aluminium substrates in oxalic acid [26]. Sustaining aluminium was removed using a $\mathrm{CuCl}_{2} / \mathrm{HCl}$ solution. The oxide barrier layer was opened by chemical etching, exposing the backside of the membranes to a $5 w t . \% \mathrm{H}_{3} \mathrm{PO}_{4}$ solution during $120 \mathrm{~min}$. During this time, the pores open and channels wide from $35 \mathrm{~nm}$ to $52 \mathrm{~nm}$ in diameter. To deposit the nickel, a metallic gold electrode was sputtered and then electrodeposited on one side of the opened pores $[11,27]$. Direct current electrodeposition of nickel was carried out at room temperature (RT) in a conventional three-electrode cell by employing an aqueous electrolyte containing $300 \mathrm{~g} / 1 \mathrm{NiSO}_{4} \bullet 6 \mathrm{H}_{2} \mathrm{O}$, $45 \mathrm{~g} / 1 \mathrm{NiCl}_{2} \bullet 6 \mathrm{H}_{2} \mathrm{O}$ and $45 \mathrm{~g} / \mathrm{l} \mathrm{H}_{3} \mathrm{BO}_{3}$, with $\mathrm{pH}$ adjusted to around 4 by dropping diluted $\mathrm{NaOH}$. A voltage of $-1.2 \mathrm{~V}$

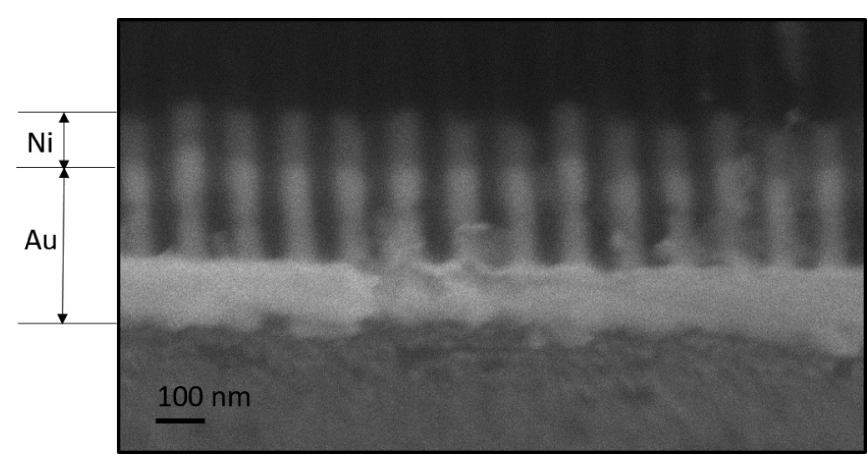

FIG. 1. Cross-sectional view of nickel NP array.

was applied with respect on the $\mathrm{Ag} / \mathrm{AgCl}$ reference electrode. Deposition time was only $5 \mathrm{~s}$ in order to produce low aspect ratio particles. Cross-sectional view SEM images of the NPs array fabricated were simply collected by specimen breaking on a Hitachi S-4800 field-emission scanning electron microscope (FE-SEM). We observe the nickel NPs standing on the gold grown into the pore channels (see Fig. 1). The NPs are characterized by a mean diameter $(D)$ and a mean length $(L)$ of $52 \pm 2 \mathrm{~nm}$ and $100 \pm 6 \mathrm{~nm}$, respectively. The aspect ratio, $\lambda$, of the NPs formed reads $\lambda=L / D=1.9 \pm 0.1$. The NPs are arranged in a hexagonal array, where the center-to-center NP distance, $d$, is $110 \pm 3 \mathrm{~nm}$ (see Fig. 2, inset). Hereafter, we will call "specimen" the slab that circumscribes the NP array.

\section{MAGNETIC BEHAVIOR}

Magnetic moment $(m)$ as a function of applied magnetic field $(H)$ at RT was measured using a vibrating sample magnetometer (VSM) LakeShore 7404, operated with maximum applied field $\mu_{0} H_{\max }=1.9 \mathrm{~T}$. The measurements were performed on a quasisquare sample piece of approximately

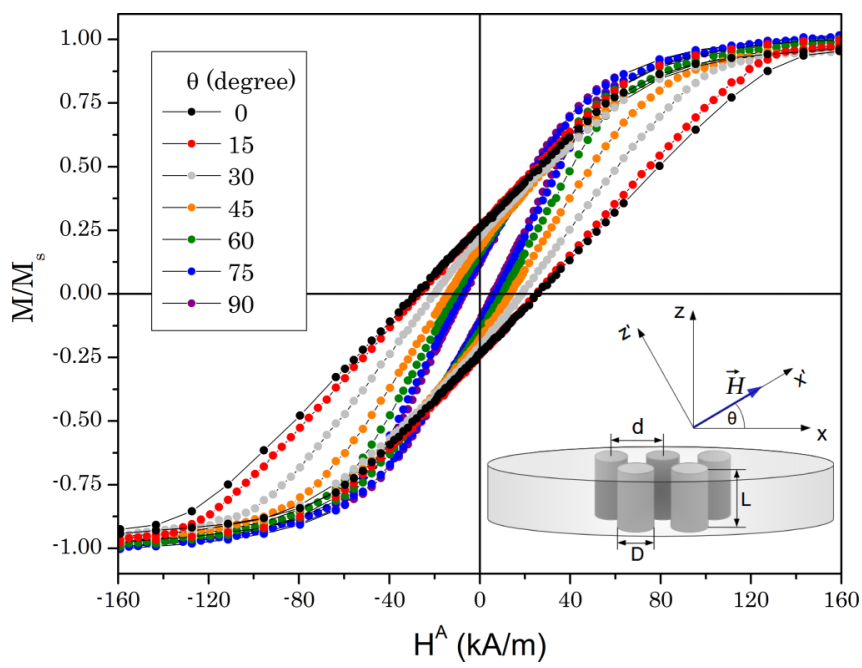

FIG. 2. Hysteresis loops measured at different angles, $\theta$, of the applied magnetic field. Inset: diagram of the NP arrangement in the alumina template and their parametrization scheme. $\theta$ is the angle between the applied field direction $v$ and an axis parallel to the alumina membrane $x$ contained in the plane $z v$, where $z$ is parallel to the NP main axes. 


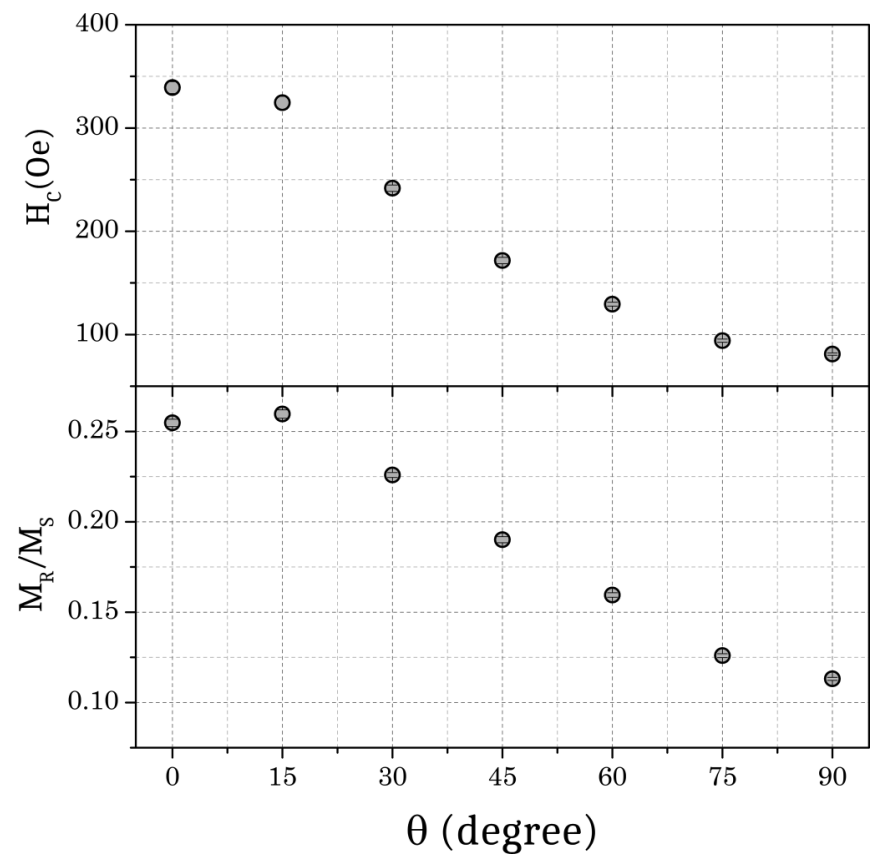

FIG. 3. Coercive field and normalized remanent magnetization values extracted from hysteresis loops measured at different angles of the applied magnetic field.

$2 \mathrm{~mm}$ per side. The measurements of the cycles began with the maximum applied field, sufficient to saturate the sample. Each cycle was performed in a range from positive $(1.9 \mathrm{~T})$ to negative saturation $(-1.9 \mathrm{~T})$ using an average rate of $10.5 \mathrm{Oe} / \mathrm{s}$. The VSM measurement time was approximately $20 \mathrm{~s}$ (each cycle contains 384 measurements). Magnetization was measured collinear to the applied magnetic field at different angles with respect to the membrane plane, by physically rotating the sample. The angle $\theta$ is defined by the field direction $(v)$ and the axis $(x)$ being the axis given by the intersection of the membrane plane and the plane $z v$, where the $z$ axis is parallel to the NPs' main axes (see Fig. 2, inset). Figure 2 shows the angular dependence of the magnetization hysteresis loops. Because of the impossibility of quantifying the total nickel mass in the sample to calculate the magnetization, in Fig. 2 is represented the normalized magnetic moment $\left(m_{n}\right)$, i.e., the measured magnetic moment divided by its highest value $\left(m_{s}\right)$. As the nickel NPs are of high quality and their ferromagnetic response is saturated at the higher applied field, the obtained $m_{n}=m / m_{s}$ should be very close to the normalized magnetization $M_{n}=M / M_{s}$, where $M$ is the magnetization and $M_{s}$ is the nickel saturation magnetization. The coercive field $\left(H_{c}\right)$ and the remanence normalized by the saturation value $\left(M_{R} / M_{S}\right)$, as a function of $\theta$, are shown in Fig. 3. Normalized remanences are smaller than 0.26 (Fig. 3, bottom), thus indicating a particlelike behavior, i.e., far from the high remanences reported for high aspect ratio nanowires [28]. Qualitatively, taking into account the values of the curve slopes near the coercive field, an easy axis normal to the plane, i.e., parallel to the NP axes, can be inferred. In this respect, note the increase of coercivity towards the plane (Fig. 3, top), which has been reported as a clear signature of dipolar interactions among the nanopillars [29,30].

\section{EFFECTIVE DEMAGNETIZING TENSOR MODEL}

The magnetic susceptibility is a quantity that describes the capability of a material to be magnetized by application of a magnetic field $\vec{H}$. It depends on the initial magnetization state $\vec{M}$ of the sample. In general, as the response can occur in other direction than the applied field one, it has to be represented by a second rank tensor. This tensor character of the susceptibility is related to the anisotropy of the magnetic material. The component $\chi_{i j}$ of the differential susceptibility tensor $\chi$ describes the magnetization variation in the $i$ th direction from an incremental change in the $j$ th direction of the applied field. Its $3 \times 3$ matrix components are defined as $\chi_{i j}=\frac{\partial M_{i}}{\partial H_{j}}$. By an appropriate choice of coordinate system, the susceptibility tensor can be reduced to three independent components in a diagonal form. The diagonalization of the susceptibility tensor takes place when the privileged directions of the magnetization become parallel to the chosen coordinate system. In our case, the susceptibility becomes diagonal in the coordinate system where $x$ is parallel to the plane of the alumina membrane and $z$ parallel to the NP main axis. Owing to the cylindrical geometry of the NPs, there are only two independent components, reducing the susceptibility to a $2 \times 2$ matrix:

$$
\chi=\left[\begin{array}{cc}
\chi_{x x} & 0 \\
0 & \chi_{z z}
\end{array}\right]
$$

Due to magnetostatic interactions between NPs, the effective average field $\vec{H}^{E}$ inside the sample exposed to an external applied magnetic field $\vec{H}^{A}$ is affected by a demagnetizing field $\vec{H}^{D}$ in the manner $\vec{H}^{E}=\vec{H}^{A}+\vec{H}^{D}$. The demagnetizing field is defined proportional and opposite to the magnetization. It has the form $\vec{H}^{D}=-N^{E} \vec{M}$, where $N^{E}$ is the effective demagnetizing tensor. Thus, the components of the measured susceptibility, hereinafter called apparent susceptibility tensor $\kappa, \kappa_{i j}=\left.\frac{\partial M_{i}}{\partial H_{j}^{A}}\right|_{M_{i}=0}$ are different than the true susceptibility ones $\chi_{i j}=\left.\frac{\partial M_{i}}{\partial H_{j}^{E}}\right|_{M_{i}=0}$. We have designated true susceptibility to the intrinsic one, i.e., the susceptibility that NPs would have if they were noninteracting, and apparent to that of the interacting NPs in the specimen since it includes extrinsic effects determinated by their spatial distribution. The relation between them is given by:

$$
\boldsymbol{\kappa}=\left(\chi N^{E}+I\right)^{-1} \chi,
$$

where $\boldsymbol{I}$ is the identity matrix. This relationship can be obtained considering that $\vec{M}=\kappa \vec{H}^{A}=\chi \vec{H}^{E}$ (valid for small values of $M$ ) and $\vec{H}^{E}=\vec{H}^{A}-N^{E} \vec{M}$. Therefore, to determine $\chi$ from the $\boldsymbol{\kappa}$ measurement, it is necessary to know $N^{E}$. The latter depends on the NP volume fraction, the specimen shape, and the presence of spatial-distribution inhomogeneities of the NPs (clusters) [23].

From each $M$ versus $H$ hysteresis loop measured at different angles $\theta$ [defined by the field direction $(v)$ and an axis $(x)$ parallel to the membrane] presented in Fig. 2, it is possible to obtain the $\kappa_{x x}^{\prime}$ component of the matrix $\kappa^{\prime}$. The prime symbol indicates that the matrix is referred to the basis 


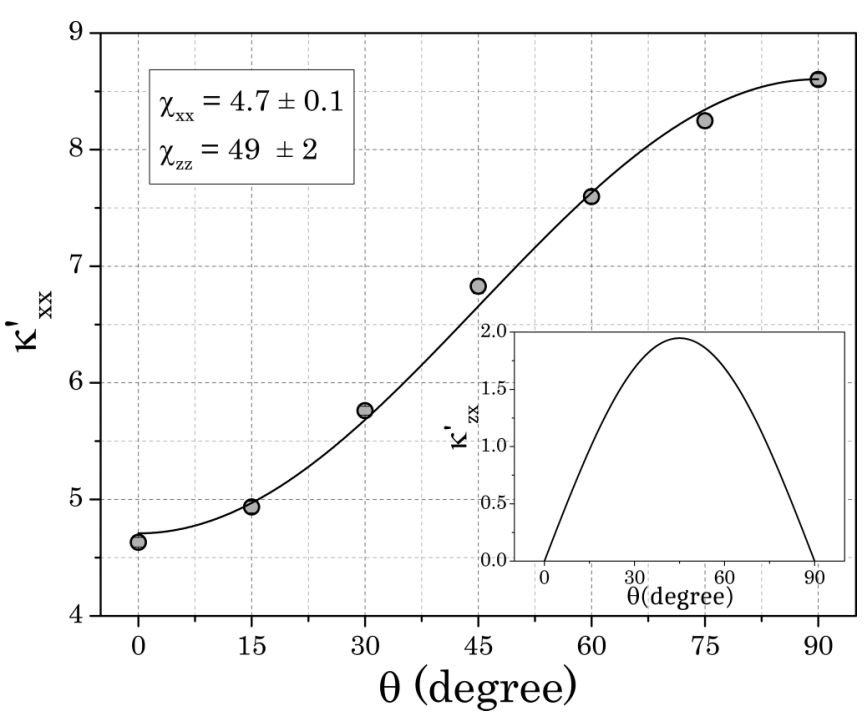

FIG. 4. Circles: Initial slope $\kappa_{x x}^{\prime}=\left.\frac{\partial M_{x}^{\prime}}{\partial H_{x}^{\prime \prime}}\right|_{M_{x}^{\prime}=0}$ extracted experimentally at different angles of the applied field. Line: Best fit with function predicted by the model, Eq. (5). Legend: True susceptibility component values recovered from the fit. Inset: Initial slope perpendicular to the magnetic field direction $\kappa_{z x}^{\prime}=\left.\frac{\partial M_{z}^{\prime}}{\partial H_{x}^{\prime A}}\right|_{M_{x}^{\prime}=0}$ obtained with the model.

$\left(x^{\prime}, z^{\prime}\right)$ where

$$
\left[\begin{array}{l}
x^{\prime} \\
z^{\prime}
\end{array}\right]=\boldsymbol{R}(\theta)\left[\begin{array}{l}
x \\
z
\end{array}\right]
$$

being

$$
\boldsymbol{R}(\theta)=\left[\begin{array}{cc}
\cos \theta & -\sin \theta \\
\sin \theta & \cos \theta
\end{array}\right]
$$

the rotation matrix (counterclockwise through an angle $\theta$ ): in consequence, $\boldsymbol{\kappa}$ reads $\boldsymbol{\kappa}^{\prime}=\boldsymbol{R}^{-1}(\theta) \boldsymbol{\kappa} \boldsymbol{R}(\theta)$. Notice that $x^{\prime}$ coincides with the field direction $v$, therefore it is possible to directly measure the $\kappa_{x x}^{\prime}$ component from the hysteresis loops in the prime basis. In other words, for small applied field values the relation $M_{x}^{\prime}=\kappa_{x x}^{\prime} H_{x}^{\prime A}+\kappa_{z z}^{\prime} H_{z}^{\prime A}$ is valid and as in the prime basis $H_{z}^{\prime A}=0$, thus $\kappa_{x x}^{\prime}=\left.\frac{\partial M_{x}^{\prime}}{\partial H_{x}^{\prime A}}\right|_{M_{x}^{\prime}=0}$. Then, the apparent susceptibility component $\kappa_{x x}^{\prime}$ is experimentally accessible from each cycle, via the magnetization initial slope $\left.\frac{\partial M_{n x}^{\prime}}{\partial H_{x}^{\prime A}}\right|_{M_{n x}^{\prime}=0}$ and the saturation magnetization as $\kappa_{x x}^{\prime}=$ $\left.4 \pi 10^{-3} \frac{\partial M_{n x}^{\prime}}{\partial H_{x}^{\prime \prime}}\right|_{M_{n x}^{\prime}=0} M_{s}$. As the preparation method ensures the formation of high-quality dense nickel NPs, and their sizes are relatively large, we can assume $M_{s} \approx 4.908 \times 10^{5} \mathrm{~A} / \mathrm{m}$, the bulk nickel saturation magnetization [31]. The $\kappa_{x x}^{\prime}$ values extracted from the loops measured at the different angles are shown in Fig. 4. The values of $\kappa_{x x}^{\prime}$ obtained at $\theta=0^{\circ}$ and $\theta=90^{\circ}$ correspond to susceptibilities in the principal directions $x$ and $z, \kappa_{x x}$ and $\kappa_{z z}$, respectively. Their values in SI units are $\kappa_{x x}=4.63 \pm 0.04$ and $\kappa_{z z}=8.60 \pm 0.05$, respectively. These values reveal that the easy magnetization axis is normal to the sample plane, i.e., parallel to the NP axes.

Let us consider quantitatively these results in relation to a simple isolated pillar without magnetostatic interactions. To estimate the magnetic anisotropy energy $K$, we have consid- ered two main contributions, crystalline $K_{N i}$ and magnetostatic (shape) $K_{\mathrm{ME}}$ anisotropies. The magnetostatic anisotropy can be calculated as: $K_{\mathrm{ME}}=\frac{\mu_{0}}{2}\left(N_{x x}-N_{z z}\right) M_{s}^{2}$. For nickel $K_{N i} \approx 4.5 \times 10^{3} \mathrm{~J} / \mathrm{m}^{3}$ [32] and for a cylinder with an aspect ratio $\lambda=1.9, N_{z z}=0.188$ and $N_{x x}=0.406$ [33], resulting in $K_{\mathrm{ME}}=3.3 \times 10^{4} \mathrm{~J} / \mathrm{m}^{3}$, almost one order of magnitude higher than the crystalline one. Therefore, the magnetic anisotropy is dominated by internal magnetostatic effects. To proceed further we must explore if NPs can be safely considered single-domain particles. The NP magnetic behavior depends on (i) the magnetic exchange length $\xi_{e}=\sqrt{\frac{4 \pi A}{\mu_{0} M_{s}^{2}}}$, where $A$ is the exchange stiffness parameter, and (ii) on the aspect ratio $\lambda$ [34]. For a cylinder with an aspect ratio $\lambda=1.9$, the critical diameter $D_{\mathrm{CR}}$ below which the nanoparticles are single domain is $D_{\mathrm{CR}} \approx 3.6 \xi_{e}$ [32]. For nickel, $A=1 \times 10^{-11} \mathrm{~J} / \mathrm{m}$, therefore $\xi_{e}=21 \mathrm{~nm}$ and the critical diameter becomes $75 \mathrm{~nm}$. Thus, the NPs considered in this work, with diameters of $52 \pm 2 \mathrm{~nm}$, can be assumed as single-domain. Therefore, the reduced anisotropy reads $\sigma=\frac{V K_{\mathrm{ME}}}{k_{B} T}=2.2 \times 10^{3}$, where $V$ is the pillar volume, $k_{B}$ the Boltzmann constant and $T$ the temperature (RT in this case). As $\sigma \gg 1$, a Stoner-Wohlfarth-like [35] behavior is expected with $\chi_{z z} \rightarrow \infty$ and $\chi_{x x} \approx \frac{\mu_{0} M_{s}^{2}}{2 K_{\mathrm{ME}}} \approx 4.6$. The fact that $\chi_{x x} \approx \kappa_{x x}$ but $\chi_{z z} \gg \kappa_{z z}$ indicates that dipolar interaction effects are anisotropic.

To determine the intrinsic properties of the NPs, as $\chi$, in presence of dipolar interactions and demagnetizing effects, it is necessary to use a model that describes these phenomena. Recently, an expression for the principal components of $N^{E}$ was developed and tested within the frame of a mean-field interacting superparamagnet model [23]. It describes how magnetic dipolar interactions modify the response of an ensemble of single magnetic domain particles to an applied magnetic field. The model was applied to three-dimensional randomlike ensembles of nearly spherical nanoparticles in thermal equilibrium, conditions that do not require a tensor analysis, as it does in the present case. It describes the dipolar field generated by the particles, which may be aggregated in clusters, by means of an effective demagnetizing tensor $N^{E}$, when the magnetic field is applied in a principal direction $u$ of the sample. This diagonal tensor is a simple function of demagnetizing tensors associated with specimen shape $\boldsymbol{N}^{s}$ and mean cluster shape $\boldsymbol{N}^{c}$, and of the mean near-neighbor distances between nanoparticles $d$ and between clusters $d_{c}$. The so-called distances are relative to the characteristic sizes of each of these two types of objects, $D$ and $D_{c}$ respectively. The demagnetizing factor in the $u$ direction reads:

$$
N_{u u}^{E}=\frac{\varphi}{\gamma^{3}}\left[N_{u u}^{c}\left(1-\frac{\varphi_{c}}{\gamma_{c}^{3}}\right)+N_{u u}^{s} \frac{\varphi_{c}}{\gamma_{c}^{3}}\right],
$$

where $\gamma=\frac{d}{D}\left(\gamma_{c}=\frac{d_{c}}{D_{c}}\right)$ is the mean relative distance for nanoparticles (clusters) and $\varphi\left(\varphi_{c}\right)$ is the volume ratio associated to all particles (clusters) within a cluster (the specimen) to the cluster (specimen) volume. In the present paper, due to the uniform distribution of the NPs in the alumina template, the presence of clusters has not been considered. Thus, both $\varphi_{c}$ and $\gamma_{c}$ are equal to unity and Eq. (3) simplifies to:

$$
N_{u u}^{E}=\frac{\varphi}{\gamma^{3}} N_{u u}^{s} .
$$


$N^{E}$ maintains the inverse cubic dependence on NP separation, characteristic of dipolar interactions. It also ensures that when specimen becomes a magnetic continuum, i.e., $\varphi$ and $\gamma$ equal to unity, $\boldsymbol{N}^{E}=\boldsymbol{N}^{s}$; while $\boldsymbol{N}^{E} \rightarrow 0$ for $\gamma \rightarrow \infty$, leaving the NPs intrinsic response unchanged in the last case. For the particular case of our two-dimensional array of ordered cylinders of diameter $D$ in a hexagonal array of lattice parameter $d$, we redefine $\gamma=\frac{d}{D}$. On the other hand, $\varphi$ is the maximum possible volume fraction of NPs in the specimen, i.e., the one corresponding to the NPs in mutual contact [23]. It can be easily calculated as $\varphi=\frac{\pi}{4 \cos \frac{\pi}{6}} \approx 0.907$. Considering the shape of the specimen, its demagnetizing factors in the principal directions are $N_{x x}^{s} \approx 0$ and $N_{z z}^{s} \approx 1$. Thus, $N_{z z}^{E}=\frac{\varphi}{\gamma^{3}} \approx 0.096$ while $N_{x x}^{E}$ obviously equals to zero.

Once $N^{E}$ is known, it is possible to obtain $\vec{H}^{E}=\vec{H}^{A}+$ $\vec{H}^{D}=\vec{H}^{A}-N^{E} \vec{M}$ along the principal directions. Thus, $M_{z}$ can be plotted as a function of $H_{z}^{E}$ for each value of the applied field. $H_{z}^{E}$ was calculated using the value of $N_{z z}^{E}=0.096$ from Eq. (4). For the sake of comparison, in Fig. 5, $M_{z}$ is presented versus the applied $\left(H_{z}^{A}\right)$ and effective $\left(H_{z}^{E}\right)$ fields at $\theta=90^{\circ}$. We can visually observe, as the deshearing of the hysteresis loop [9], the recovering of $\chi_{z z}$ from its apparent value $\kappa_{z z}$, being in this case $\chi_{z z}>\kappa_{z z}$.

$$
\boldsymbol{\kappa}^{\prime}=\left[\begin{array}{l}
\frac{\chi_{x x}}{1+N_{x x}^{E} \chi_{x x}} \cos ^{2} \theta+\frac{\chi_{z z}}{1+N_{z z}^{E} \chi_{z z}} \sin ^{2} \theta \\
\left(\frac{\chi_{z z}}{1+N_{z z}^{E} \chi_{z z}}-\frac{\chi_{x x}}{1+N_{x x}^{E} \chi_{x x}}\right) \cos \theta \sin \theta
\end{array}\right.
$$

Using Eq. (5) with $N_{x x}^{E}=0$ and $N_{z z}^{E}=\frac{\varphi}{\gamma}$, the experimental value of $\kappa_{x x}^{\prime}$ was fitted. From the fit, the components of $\chi$ were recovered, obtaining $\chi_{x x}=4.7 \pm 0.1$ and $\chi_{z z}=49 \pm 2$ (see Fig. 4). These values are in agreement with the StonerWohlfarth model predictions, i.e., $\chi_{z z} \gg \chi_{x x}$ and $\chi_{x x} \approx 4.6$. The finite value of $\chi_{z z}=49 \pm 2$, instead of the infinite value predicted by the Stoner-Wohlfart model, is due to the fact that temperature is not strictly zero and NP main axes would not be perfectly perpendicular to the plane of the alumina membrane, but they would present stochastic misalignments.

After determining the components of $\chi$, it is possible to estimate the $\kappa_{z x}^{\prime}$ component, which is related to the magnetization perpendicular to the applied magnetic field. This magnetization component arises from the anisotropies of the sample as a whole (extrinsic effects) and of the NPs (intrinsic effects). The result is shown in Fig. 4, inset. As it was expected, when the field is applied along a nonprincipal direction, $\vec{M}$ has a component perpendicular to $\vec{H}^{A}$. It is also possible to obtain the components of $\chi^{\prime}$ as:

$$
\chi^{\prime}=\left[\begin{array}{ll}
\chi_{x x} \cos ^{2} \theta+\chi_{z z} \sin ^{2} \theta & \left(\chi_{z z}-\chi_{z z}\right) \cos \theta \sin \theta \\
\left(\chi_{z z}-\chi_{z z}\right) \cos \theta \sin \theta & \chi_{z z} \cos ^{2} \theta+\chi_{x x} \sin ^{2} \theta
\end{array}\right]
$$

$\chi_{x x}^{\prime}, \chi_{z z}^{\prime}$, and $\chi_{x z}^{\prime}$ are represented in Fig. 6 as a function of the measurement angle $\theta$. The knowledge of all components of $\chi$ results in a complete characterization of the magnetic response of a nanopillar under a low applied magnetic field

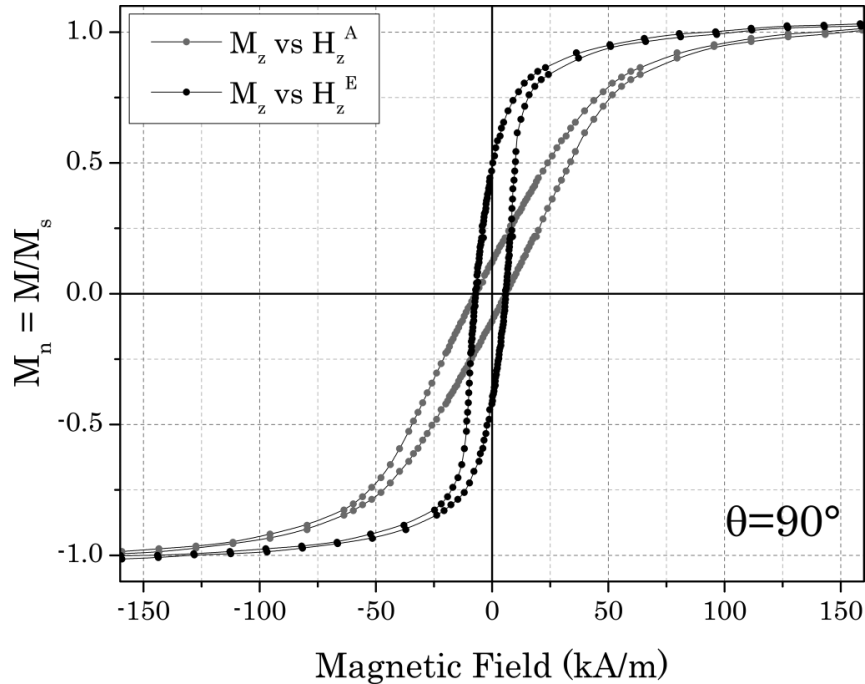

FIG. 5. Experimental dependence of $M_{z}$ versus the applied $\left(H_{z}^{A}\right)$ and effective $\left(H_{z}^{E}\right)$ fields at $\theta=90^{\circ} . H_{z}^{E}$ was calculated using $N_{z z}^{E}=$ 0.096 from Eq. (4).

Once $\boldsymbol{N}^{E}$ is determined, it is possible to obtain an expression of $\boldsymbol{\kappa}^{\prime}$ in terms of the components of $\boldsymbol{\chi}$ :

$$
\left.\begin{array}{l}
\left(\frac{\chi_{z z}}{1+N_{z z}^{E} x_{z z}}-\frac{\chi_{x x}}{1+N_{x x}^{E} x_{x x}}\right) \cos \theta \sin \theta \\
\frac{\chi_{x x}}{1+N_{x x}^{E} \chi_{x x}} \cos ^{2} \theta+\frac{x_{z z}}{1+N_{z z}^{E} \chi_{z z}} \sin ^{2} \theta
\end{array}\right] .
$$

in any direction. In addition, the non-null values of the tensor component $\chi_{z x}$, revealed in Fig. 6, highlight the need of using the tensor form of the susceptibility.

Finally, for low applied fields, thus supposing a linear response regime, the angle $\alpha$ between the sample plane and the magnetization increment $\Delta \vec{M}$, corresponding to an applied

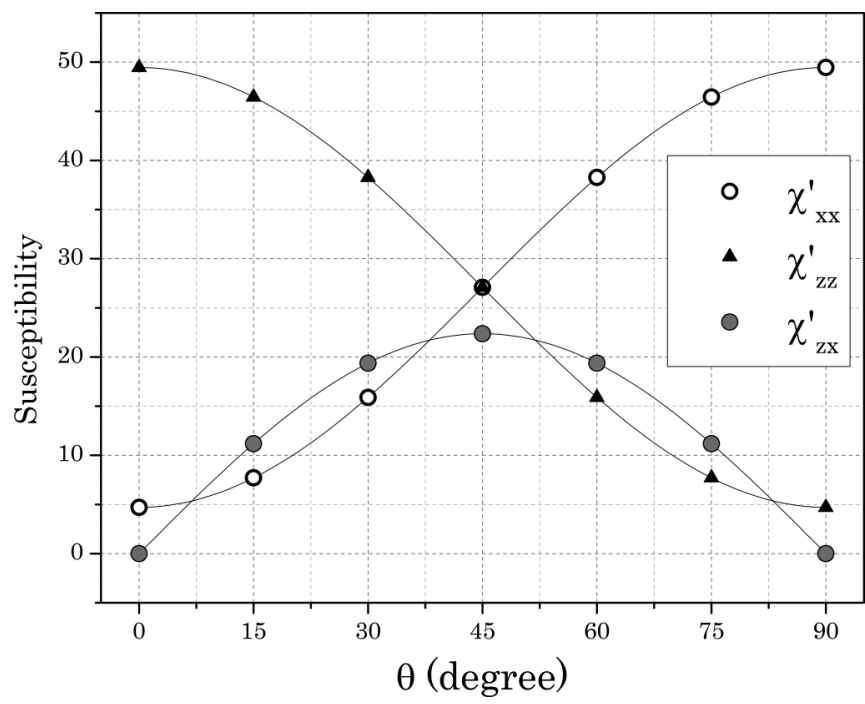

FIG. 6. $\chi_{x x}^{\prime}, \chi_{z z}^{\prime}$, and $\chi_{x z}^{\prime}$ components as a function of the angle $\theta$ between magnetic field and $x$ axis (parallel to the plane of the alumina membrane). 


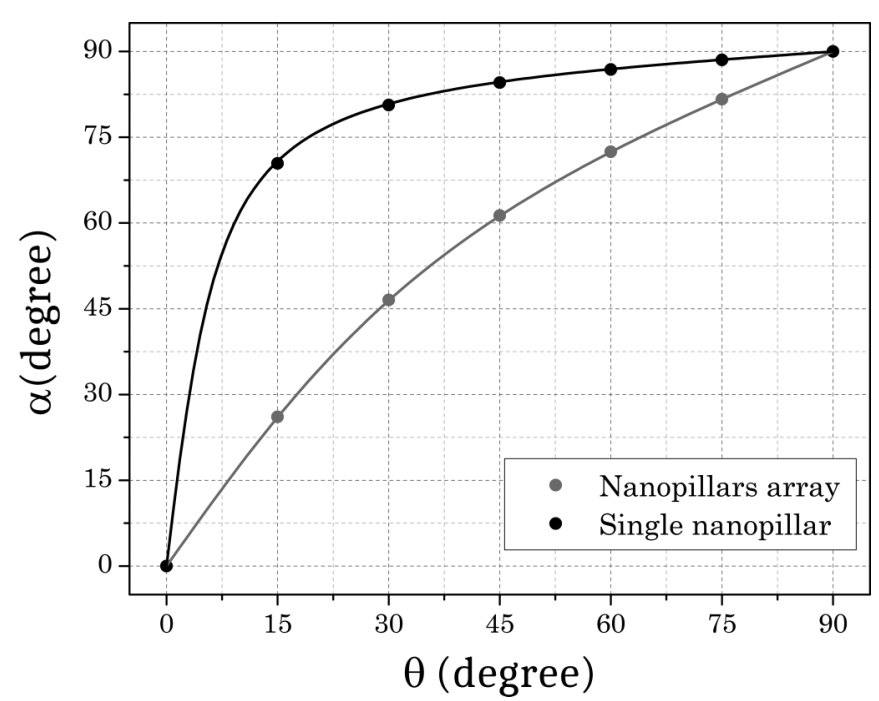

FIG. 7. Angle $\alpha$ between magnetization increment $\Delta \vec{M}$ and $x$ axis (parallel to the plane of the alumina membrane) for low magnetic fields. The NP array and single NP (without dipolar interactions) results are compared.

field increment $\Delta \vec{H}^{A}$, is

$$
\tan \alpha=\frac{1+N_{x x}^{E}}{1+N_{z z}^{E}}\left(\frac{\chi_{z z}}{\chi_{x x}}\right) \tan \theta .
$$

This $\alpha$ angle is presented in Fig. 7 as a function of $\theta$. It is compared with the values that correspond to an isolated NP (without dipolar interactions). We observe that $\alpha \neq \theta$ for the present case as it would be expected for a general anisotropic situation. Figure 7 shows how dipolar interaction between NPs strongly affects the magnetic response of the NP ensemble. For example, a low magnetic field applied over the NP ensemble in a direction with angle $\theta=15^{\circ}$ (with respect to the membrane plane) results in a magnetization direction of $\alpha=25^{\circ}$; however, if this field is applied on a single NP, the magnetization direction will be at $\alpha=70^{\circ}$. The most general situation in which $\alpha=\theta$ for any $\theta$ value would be an isotropic spatial distribution of isotropic or randomly oriented anisotropic particles. In that case, $N_{x x}^{E}=N_{z z}^{E}$ and $\left\langle\chi_{x x}\right\rangle=\left\langle\chi_{z z}\right\rangle$, where \langle\rangle stands for the mean value obtained over all particles of the ensemble.

\section{SUMMARY AND CONCLUSIONS}

A high-quality two-dimensional hexagonal centered arrangement of low aspect ratio nickel parallel NPs, characterized by mean values of diameter $D=52 \pm 2 \mathrm{~nm}$, length $L=100 \pm 6 \mathrm{~nm}$, and lattice constant $d=110 \pm 3 \mathrm{~nm}$, was fabricated via dc electrodeposition within the nanopores of a porous alumina membrane. Magnetic moment vs. applied field hysteresis loops were measured on this specimen as a function of the angle $\theta$ between the membrane plane and the applied magnetic field. The angular dependence of the coercivity, the remanent magnetization and the low field slope $\left.\frac{\partial M_{i}^{\prime}}{\partial H_{i}^{\prime A}}\right|_{M_{i}^{\prime}=0}$, point that $\theta=90^{\circ}$ is the easy magnetization direction, i.e., parallel to the NP axes.

According to their aspect ratio $\lambda=L / D=1.9 \pm 0.1$, the NPs should be single domain for $D<75 \mathrm{~nm}$, a condition fulfilled in the present case [34]. Thus, the magnetostatic anisotropy $K_{\mathrm{ME}}=\frac{\mu_{0}}{2}\left(N_{x x}-N_{z z}\right) M_{s}^{2}=3.3 \times$ $10^{4} \mathrm{~J} / \mathrm{m}^{3}$ dominates, and leads to a reduced anisotropy $\sigma=\frac{V K_{\mathrm{ME}}}{k_{B} T}=2.2 \times 10^{3}$, which indicates that an isolated pillar should respond approximately according to the StonerWohlfarth model. In fact, the values obtained for the nanopillar susceptibility $\chi$ from the EDT model $\left(\chi_{x x}=4.7 \pm 0.1\right.$ and $\chi_{z z}=49 \pm 2$ ) fulfill that $\chi_{z z} \gg \chi_{x x}$ and $\chi_{x x} \approx \frac{\mu_{0} M_{s}^{2}}{2 K_{\mathrm{ME}}}$.

The EDT model recently reported [23] was adapted to the present situation, i.e., a two-dimensional ordered arrangement of identical pillars. The adopted EDT form is simple [Eq. (4)], only depending on three parameters: the near-neighbor distance between pillars relative to pillar diameter, the associated volume fraction of pillars in the specimen, and the demagnetizing tensor corresponding to the specimen shape. Along principal directions $u$, the EDT has the form $N_{u u}^{E}=\frac{\varphi}{\gamma^{3}} N_{u u}^{s}$, being $N_{x x}^{s}=0$ and $N_{z z}^{s}=1$. Once the EDT is known, Eq. (2) allows the experimental determination of the true susceptibility $\chi$ from the apparent $\kappa$, obtained from conventional magnetization measurements. From the measured $\kappa_{x x}^{\prime}$ tensor component in a reference frame bound to the applied field direction $\left(x^{\prime}\right)$, the tensors $\boldsymbol{\kappa}, \boldsymbol{\chi}, \boldsymbol{\kappa}^{\prime}$, and $\chi^{\prime}$ were determined. It was demonstrated that for $0^{\circ}<\theta<90^{\circ}$ the magnetization has a component normal to the applied field, which is revealed by the non-null values of the tensor elements $\chi_{z x}^{\prime}$ and $\kappa_{z x}^{\prime}$.

This paper demonstrates that this simple EDT model can be applied in very different ordering conditions: from ensembles of nanoparticles characterized by size, position, and orientation randomness [23], to high ordered arrangements of quasi-identical objects. In the last case, by means of a simple morphological characterization of the ensemble, the intrinsic response of the susceptibility at any direction can be extracted in an easy and rapid way. Thus, the model should be useful to design nanomagnet lattices [36] or colloids [15] with tunable response. The foreseeable breakdown of the present formulation in terms of the object size to interobject distances ratio for very anisotropic objects such as micrometer-long nanowires still needs to be evaluated.

The EDT model highlights the importance of the sample geometry and the nanoparticle ordering in the response. None of the models applied up to now to the analysis of experimental results, where interparticle dipolar interactions play a relevant role, has considered these geometrical aspects, and this may well be the cause of why important experimental information is frequently omitted in scientific reports. We expect that the successful EDT application to the susceptibility of out-of-equilibrium interacting nanoparticles will lead to a relationship in which the global relaxation time will be associated to that corresponding for a noninteracting system. In conclusion, the actual formulation of the EDT model provides an approximated treatment of the complicated issue of dipolar interactions in a simple and accurate manner, even in anisotropic and out-of-equilibrium discrete systems, allowing the inference of relevant physical information.

\section{ACKNOWLEDGMENTS}

This research has been funded under CONICET Grant No. PIP 11220110100720CO and UNLP Grant No. 11/X680, both from Argentina; FAPESP Grants No. 2012/04870-7 
and No. 2013/13275-8 from Brazil; and MINECO projects MAT2013-48054-C2-2-R and MAT2016-76824-C3-3-R from Spain. We acknowledge the technical support from the
Scientific-Technical Services of the University of Oviedo (SCT's) and particularly to the Laboratory of Nanoporous Membranes.
[1] J. L. Dormann, D. Fiorani, and E. Tronc, Magnetic Relaxation in Fine-Particle Systems, Volume 98 of Advances in Chemical Physics, edited by I. Prigogine and S. A. Rice (Wiley, New York, 1997), pp. 283-494.

[2] S. Bedanta and W. Kleemann, Supermagnetism, J. Phys. D 42, 013001 (2009).

[3] R. López-Ruiz, F. Luis, J. Sesé, J. Bartolomé, C. Deranlot, and F. Petroff, Zero-temperature spin-glass freezing in self-organized arrays of Co nanoparticles, Europhys. Lett. 89, 67011 (2010).

[4] G. A. Badini Confalonieri, V. Vega, A. Ebbing, D. Mishra, P. Szary, V. M. Prida, O. Petracic, and H. Zabel, Templateassisted self-assembly of individual and clusters of magnetic nanoparticles, Nanotechnol. 22, 285608 (2011).

[5] K. Nielsch, R. B. Wehrspohn, J. Barthel, J. Kirschner, U. Gosele, S. F. Fischer, and H. Kronmuller, Hexagonally ordered 100 nm period nickel nanowire arrays, Appl. Phys. Lett. 79, 1360 (2001).

[6] H. He and N. J. Tao, Electrochemical Fabrication of Metal Nanowires, Volume 2 of Encyclopedia of Nanoscience and Nanotechnology, edited by H. S. Nalwa (American Scientific, Valencia, 2004), Chap. 34, pp. 755-772.

[7] J. García, V. Vega, L. Iglesias, V. M. Prida, B. Hernando, E. D. Barriga-Castro, R. Mendoza-Reséndez, C. Luna, D. Görlitz, and K. Nielsch, Template-assisted Co-Ni alloys and multisegmented nanowires with tuned magnetic anisotropy, Phys. Status Solidi A 211, 1041 (2014).

[8] G. Kartopu, O. Yalçin, M. Es-Souni, and A. C. Basaran, Magnetization behavior of ordered and high density Co nanowire arrays with varying aspect ratio, J. Appl. Phys. 103, 093915 (2008).

[9] P. M. Paulus, F. Luis, M. Kröll, G. Schmid, and L. J. de Jongh, Low-temperature study of the magnetization reversal and magnetic anisotropy of $\mathrm{Fe}, \mathrm{Ni}$, and Co nanowires, J. Magn. Magn. Mater. 224, 180, (2001).

[10] V. Vega, V. M. Prida, J. A. García, and M. Vázquez, Torque magnetometry analysis of magnetic anisotropy distribution in Ni nanowire arrays, Phys. Status Solidi A 208, 553 (2011).

[11] V. Vega, T. Böhnert, S. Martens, M. Waleczek, J. M. MonteroMoreno, D. Görlitz, V. M. Prida, and K. Nielsch, Tuning the magnetic anisotropy of Co-Ni nanowires: comparison between single nanowires and nanowire arrays in hard-anodic aluminum oxide membranes, Nanotechnol. 23, 465709 (2012).

[12] P. Sergelius, J. Garcia Fernandez, S. Martens, M. Zocher, T. Böhnert, V. Vega Martinez, V. M. Prida, D. Görlitz, and K. Nielsch, Statistical magnetometry on isolated NiCo nanowires and nanowire arrays: A comparative study, J. Phys. D 49, 145005 (2016).

[13] N. Eibagi, J. J. Kan, F. E. Spada, and E. E. Fullerton, Role of dipolar interactions on the thermal stability of high-density bit-patterned media, IEEE Magn. Lett. 3, 4500204 (2012).

[14] G. T. Landi, Role of dipolar interaction in magnetic hyperthermia, Phys. Rev. B 89, 014403 (2014).

[15] D. F. Coral, P. Mendoza Zélis, M. Marciello, M. P. Morales, A. Craievich, F. H. Sánchez, and M. B. Fernández van Raap, Effect of nanoclustering and dipolar interactions in heat generation for magnetic hyperthermia, Langmuir 32, 1201 (2016).

[16] J. M. Martínez Huerta, J. De La Torre Medina, L. Piraux, and A. Encinas, Self consistent measurement and removal of the dipolar interaction field in magnetic particle assemblies and the determination of their intrinsic switching field distribution, J. Appl. Phys. 111, 083914 (2012).

[17] A. Hillion, A. Tamion, F. Tournus, C. Albin, and V. Dupuis, From vanishing interaction to superferromagnetic dimerization: Experimental determination of interaction lengths for embedded co clusters, Phys. Rev. B 95, 134446 (2017).

[18] C. R. Pike, A. P. Roberts, and K. L. Verosub, Characterizing interactions in fine magnetic particle systems using first order reversal curves, J. Appl. Phys. 85, 6660 (1999).

[19] A. N. Dobrynin, T. R. Gao, N. M. Dempsey, and D. Givord, Experimental determination of the magnetization dependent part of the demagnetizing field in hard magnetic materials, Appl. Phys. Lett. 97, 192506 (2010).

[20] A. Berger, Y. Xu, B. Lengsfield, Y. Ikeda, and E. E. Fullerton, Delta/H(M,/spl Delta/M) method for the determination of intrinsic switching field distributions in perpendicular media, IEEE Trans. Magn. 41, 3178 (2005).

[21] I. Tagawa and Y. Nakamura, Relationships between high density recording performance and particle coercivity distribution, IEEE Trans. Magn. 27, 4975 (1991).

[22] T. Wang, Y. Wang, Y. Fu, T. Hasegawa, H. Oshima, K. Itoh, K. Nishio, H. Masuda, F. S. Li, H. Saito, and S. Ishio, Magnetic behavior in an ordered co nanorod array, Nanotechnol. 19, 455703 (2008).

[23] F. H. Sánchez, P. Mendoza Zélis, M. L. Arciniegas, M. B. G. A. Pasquevich, and F. van Raap, Dipolar interaction and demagnetizing effects in magnetic nanoparticle dispersions: Introducing the mean-field interacting superparamagnet model, Phys. Rev. B 95, 134421 (2017).

[24] G. T. Landi, F. R. Arantes, D. R. Cornejo, A. F. Bakuzis, I. Andreu, and E. Natividad, Ac susceptibility as a tool to probe the dipolar interaction in magnetic nanoparticles, J. Magn. Magn. Mater. 421, 138 (2017).

[25] P. Allia, M. Coisson, T. Paola, F. Vinai, M. Knobel, M. Novak, and W. Nunes, Granular $\mathrm{Cu}-\mathrm{Co}$ alloys as interacting superparamagnets, Phys. Rev. B 64, 144420 (2001).

[26] H. Masuda and K. Fukuda, Ordered metal nanohole arrays made by a two-step replication of honeycomb structures of anodic alumina, Science 268, 1466 (1995).

[27] M. P. Proença, C. T. Sousa, J. Ventura, M. Vázquez, and J. Araujo, Distinguishing nanowire and nanotube formation by the deposition current transients, Nanoscale Res. Lett. 7, 280 (2012).

[28] R. López-Ruiz, C. Magén, F. Luis, and J. Bartolomé, High temperature finite-size effects in the magnetic properties of ni nanowires, J. Appl. Phys. 112, 073906 (2012).

[29] J. M. García, A. Asenjo, J. Velázquez, D. García, M. Vázquez, P. Aranda, and E. Ruiz-Hitzky, Magnetic behavior of an array of cobalt nanowires, J. Appl. Phys. 85, 5480 (1999). 
[30] J. Velázquez, C. García, M. Vázquez, and A. Hernando, Dynamic magnetostatic interaction between amorphous ferromagnetic wires, Phys. Rev. B 54, 9903 (1996).

[31] J. Crangle and G. M. Goodman, The magnetization of pure iron and nickel, Proc. R. Soc. London A 321, 477 (1971).

[32] H. Sato and B. S. Chandrasekhar, Determination of the magnetic anisotropy constant $K_{2}$ of cubic ferromagnetic substances, J. Phys. Chem. Solids 1, 228 (1957).

[33] R. I. Joseph, Ballistic demagnetizing factor in uniformly magnetized cylinders, J. Appl. Phys. 37, 4639 (1966).
[34] C. A. Ross, M. Hwang, M. Shima, J. Y. Cheng, M. Farhoud, T. A. Savas, H. I. Smith, W. Schwarzacher, F. M. Ross, M. Redjdal, and F. B. Humphrey, Micromagnetic behavior of electrodeposited cylinder arrays, Phys. Rev. B 65, 144417 (2002).

[35] E. C. Stoner and E. P. Wohlfarth, A mechanism of magnetic hysteresis in heterogeneous alloys, Philos. Trans. R. Soc., A 240, 599 (1948).

[36] R. P. Cowburn, A. O. Adeyeye, and M. E. Welland, Controlling magnetic ordering in coupled nanomagnet arrays, New J. Phys. 1, 16 (1999). 